\title{
Design and Parametric Simulation of a Miniaturized PIFA Antenna for the PCS Band
}

\author{
Abdelhakim Elouadih ${ }^{1}$, Ahmed Oulad-Said ${ }^{1}$, Moha Mrabet Hassani ${ }^{2}$ \\ ${ }^{1}$ Department of Electrical and Telecommunications Engineering, Royal Air Academy (ERA), Marrakesh, Morocco; ${ }^{2}$ Department of \\ Physics, Semlalia University of Sciences (FSSM), Marrakesh, Morocco. \\ Email: elouadih@gmail.com, a_ouladsaid@hotmail.com, hassani@ucam.ac.ma
}

Received February $9^{\text {th }}, 2013$; revised March $10^{\text {th }}, 2013$; accepted March $18^{\text {th }}, 2013$

Copyright (C) 2013 Abdelhakim Elouadih et al. This is an open access article distributed under the Creative Commons Attribution License, which permits unrestricted use, distribution, and reproduction in any medium, provided the original work is properly cited.

\begin{abstract}
This paper describes the design and simulation by HFSS simulator of a probe-fed Planar Inverted-F Antenna (PIFA) for the use in PCS band [1850 MHz - $1990 \mathrm{MHz}$. A methodology based on parametric simulations (parameters are ground plan dimensions, height of radiating plate, feeding point position, shorting plate dimensions and positioning) was used to design optimized antenna. The simulation allowed the characterization of the designed antenna and the computing of different antenna parameters like $S_{11}$ parameter, resonant frequency, SWR, bandwidth impedance in feeding point, gain, $2 \mathrm{D}$ and $3 \mathrm{D}$ diagram pattern, Fields distribution. The simulation results are interesting and respect the mean PCS requirements.
\end{abstract}

Keywords: PCS; HFSS; PIFA; Patch Antenna; Parametric; Simulation

\section{Introduction}

Wireless communications have progressed very rapidly in recent years, and many mobile units are becoming smaller and smaller. To meet the miniaturization requirement, the antennas employed in mobile terminals must have their dimensions reduced accordingly. Planar antennas, such as microstrip and printed antennas have the attractive features of low profile, small size, and conformability to mounting hosts and are very promising candidates for satisfying this design consideration. Planar antennas are also very attractive for applications in communication devices for wide mobile telecommunications like GSM 1900 called PCS (Personal Communications System), wireless local area network, aeronautics and embedded systems [1].

For optimum system performance, the antennas must have high radiation efficiency, small volume, isotropic radiation characteristics, small backward radiation and SAR, simple and low-loss impedance matching to patches. The major types of configurations of low-profile antennas with enhanced bandwidth performance include planar inverted F Antennas.

The PIFA consists in general of a ground plane, a top plate element, a feed wire attached between the ground plane and the top plate, and a shorting wire or strip that is connected between the ground plane and the top plate.

The antenna is fed at the base of the feed wire at the point where the wire connects to the ground plane. The PIFA is an attractive antenna for wireless systems where the space volume of the antenna is quite limited. It requires simple manufacturing, since the radiator must only be printed. The addition of a shorting strip allows good impedance match to be achieved with a top plate that is typically less than $\lambda / 4$ long. The resulting PIFA is more compact than a conventional half-wavelength probe-fed patch antenna [2].

The miniaturization can affect radiation characteristics, bandwidth, gain, radiation efficiency and polarization purity. The miniaturization approaches are based on either geometric manipulation (the use of bend forms, meandered lines, PIFA shape, varying distance between feeder and short plate [3]) or material manipulation (loading with a high-dielectric material, lumped elements, conductors, capacitors, short plate [4]) or the environment characteristics (ground plane dimensions, coupling, measurement and fabrication errors [3]). In this case, the designed antenna is shorted to the ground plane by a plate, uses regular shapes and a high dielectric thin substrate under the radiating plate not above the ground plane.

If all precedent works are concentrated on studying the 
effects of these elements (material, geometry, environment), the choice of a PIFA element is so improvised in the design. In this paper, a methodology based on parametric simulation is used to choose simultaneously or independently different PIFA elements.

In the next section, the author presents the methodology and detailed parametric simulations to optimize the antenna design. After, the dimensions and parameters are then chosen. The author exposes the characteristics of the designed antenna in the third section.

\section{Design Methodology}

\subsection{The Description of the Studied Antenna}

As shown in Figure 1, the designed antenna has a rectangular radiating patch witch length is $L_{p}=11 \mathrm{~mm}$ and width $W_{p}=30 \mathrm{~mm}$. The patch in placed at a height $h$ from the ground plan. The last has a length $L_{g}$ and a width $W_{g}$. The patch is matched to the ground plan via a rectangular shorting plate. The shorting plate has a width $W_{s}$ and a length $L_{s}$. The shorting post of usual PIFA types is a good method for reducing the antenna size, but results in narrow impedance bandwidth. It is placed in the $(y z)$ plan at a distance $D$ from the edge centre and a distance $p$ from the feeding point. The patch is fed by a $50 \Omega$ wire; a semi-rigid coax with a centre conductor that extends beyond the end of the outer conductor is used to form the PIFA feed wire. The outer conductor of the coax is sol-

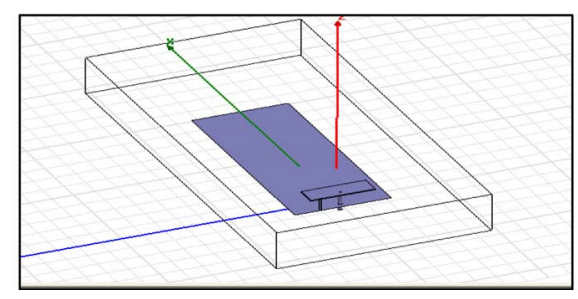

(a)

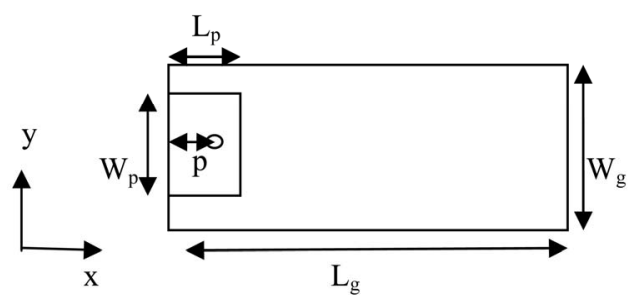

(b)

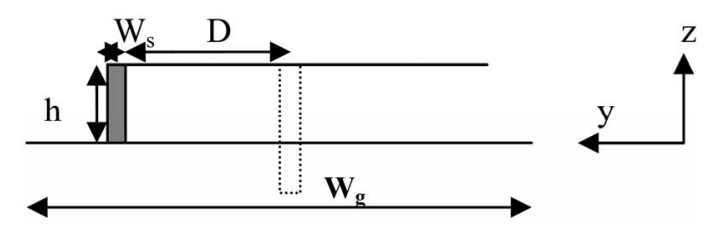

(c)

Figure 1. The designed antenna by HFSS. (a) Perspective view; (b) Top view; (c) Side view. dered to the edge of a small hole drilled in the ground plane at the feed point. The volume between the radiating plate and the ground plan is filled by air except a thin region $0.8 \mathrm{~mm}$ under the radiating patch who is composed of FR4_epoxy $\left(\varepsilon_{r}=4.4\right)$.

\subsection{The Choice of the Patch Dimensions}

It is very important for simulation by HFSS to estimate the resonant frequency that help the simulator to make a refinement mesh in a band around the resonant frequency and then give more precise values. The resonant frequency of a PIFA is approximated by the Equation (1) where $F_{r}$ is the resonant frequency, $C$ is the light velocity.

$$
F_{r}=\frac{C}{4\left(L_{p}+W_{p}-W_{s}\right)}
$$

If there is another substrate different from Air, $C$ will be $C_{0} / \sqrt{\varepsilon_{r}}$, where $C_{0}=3 \times 10^{8} \mathrm{~m} / \mathrm{s}$. For our case, the space between the patch and the ground plan is essentially air minus a $0.8 \mathrm{~mm}$ FR4 epoxy layer.

To compute the resonant frequency, we have the following values: $L_{p}=11 \mathrm{~mm}, W_{p}=30 \mathrm{~mm}, W_{s_{\min }}=1 \mathrm{~mm}$. We obtain: $F_{r}=1875 \mathrm{MHz}$.

The obtained frequency is then not far from $1920 \mathrm{MHz}$ the central frequency of PCS band. In fact, there is no equation to determine the resonant frequency for a PIFA that contains not only the patch dimensions but also the other parameters that can affect the antenna characteristics. For this, the author will make constant the patch dimensions that are the mean parameters can furnish the resonant frequency and he will vary undependably the other parameters (ground plan dimensions $L_{g}$ and $W_{g}$, height of radiating plate $h$, feeding point position $p$, shorting plate width $W_{s}$ and position $D$ ).

\subsection{The Choice of the Simulator}

HFSS (High Frequency Simulator Structure) is a high performance full wave electromagnetic (EM) field simulator for arbitrary 3D volumetric passive device modeling that takes advantage of the familiar Microsoft Windows graphical user interface. It integrates simulation, visualization, solid modeling, and automation are easy to learn environment where solutions to 3D EM problems are quickly and accurate obtained. Ansoft HFSS employs the Finite Element Method (FEM), adaptive meshing, and brilliant graphics to give unparalleled performance and insight to all of 3D EM problems. Ansoft HFSS can be used to calculate parameters such as S-Parameters, Resonant Frequency and Fields. Typical uses include Package Modeling, PCB Board Modeling, Mobile Communications (Patches, Dipoles, Horns, Conformal Cell Phone Antennas), Specific Absorption Rate (SAR), Infi- 
nite Arrays, Radar Section (RCS), Frequency Selective Surface (FSS) and filters such Cavity Filters, Microstrip, Dielectric. HFSS is an interactive simulation system whose basic mesh element is a tetrahedron T. In industry, Ansoft HFSS is the tool of choice for High productivity research, development, and virtual prototyping $[5,6]$. The HFSS is then used in our simulation; the author proposes after to expose the results of the HFSS simulations.

\subsection{The Choice of the Height $h$}

The height $h$ is the distance between the top plate and the ground plane. In order to eliminate the effects of the ground plane effects, we place the patch on the edge of an infinite ground plane at a height varying from $6 \mathrm{~mm}$ to $13 \mathrm{~mm}$. From the simulation result shown by Figure 2, the optimal height $h$ is for $h=10 \mathrm{~mm}$ because it gives values $S_{11}$ most important and closer to central frequency.

\subsection{The Choice of the Ground Plan Width $W_{g}$}

For the height equal to $10 \mathrm{~mm}$, we will vary the groundplane width by having a very important length $L_{g}$ to eliminate its effect. In general, the width $W_{g}$ is not so far to $W_{p}$ that is the minimum. For this, $W_{g}$ will vary from 30 $\mathrm{mm}$ to $60 \mathrm{~mm}$. the simulation result is shown by Figure 3. We can consider $W_{g}=45 \mathrm{~mm}$ an optimal width because it presents the minimum $S_{11}$ for all the band and

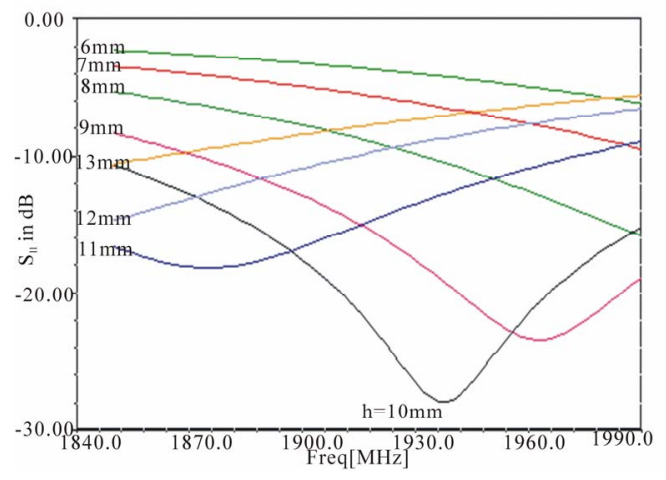

Figure 2. Parametric simulation by varying $h$.

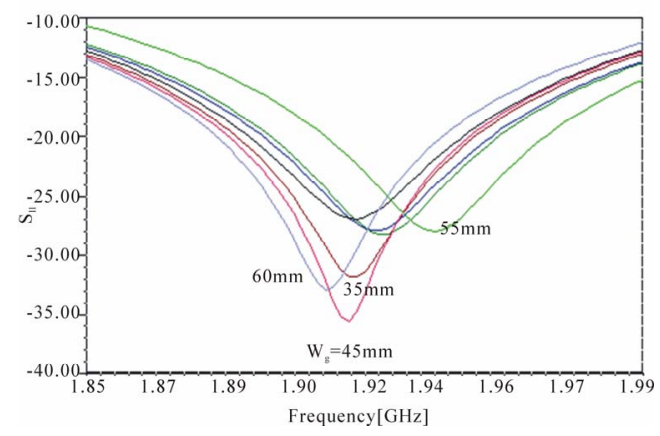

Figure 3. Parametric simulation by varying $W_{g}$. in the same time the minimum is the closest to the central frequency in the PCS band.

\subsection{The Choice of the Ground Plan Length $L_{g}$}

The height $h$ in now $10 \mathrm{~mm}$ and the $W_{g}$ is $45 \mathrm{~mm}$. We will vary the ground plane length $L_{g}$ from $60 \mathrm{~mm}$ to 140 $\mathrm{mm}$. This sweep is taken because the designed antenna will be mounted on a GSM handset. The Figure 4 shows the result of the parametric simulation. We can see from the curve that the length $100 \mathrm{~mm}$ is the most adapted. In reality, the simulation shows close results for the lengths $100 \mathrm{~mm}, 110 \mathrm{~mm}$ and $120 \mathrm{~mm}$. A sweep refinement is introduced and the optimum was $108 \mathrm{~mm}$. Also, $120 \mathrm{~mm}$ and over can be considered not interesting values for a ground plane in a handset. We have then $h=10 \mathrm{~mm}, W_{g}$ $=45 \mathrm{~mm}$ and $L_{g}=100 \mathrm{~mm}$. We will after simulate the effect of the shorting element width $W_{s}$ and distance $D$ from the patch edge centre.

\subsection{The Choice of the Shorting Plate Position $D$}

We will vary the shorting plate position between all possible values from 0 to $14 \mathrm{~mm}$, we width $W_{s}$ is in general close to $1 \mathrm{~mm}$. we obtain the result shown by Figure 5 . Both of positions 11 and $12 \mathrm{~mm}$ gibe good result. We can choose $D=11 \mathrm{~mm}$, it's closer to the central frequency.

\subsection{The Choice of the Shorting Plate Width $W_{s}$}

We will vary the width $W_{s}$ from 0.5 to $3 \mathrm{~mm}$ with step of $0.5 \mathrm{~mm}$. We will obtain the result shown by Figure 6 . We can consider the width $W_{s}=1 \mathrm{~mm}$ the most convenient for the bandwidth and the resonant frequency. From the curve, the width $W_{s}$ is close to $1 \mathrm{~mm}$. If we make a sweep refinement by choosing $0.1 \mathrm{~mm}$ step instead of 0.5 $\mathrm{mm}$ in order to hope having a $S_{11}$ peak near the central frequency if possible. We obtained the result shown by Figure 7. We can remark a net peak for the particular

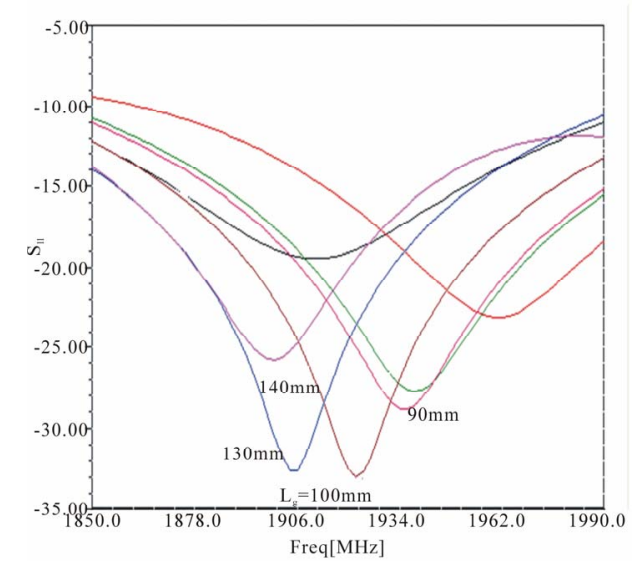

Figure 4. Parametric simulation by varying $L_{g}$. 


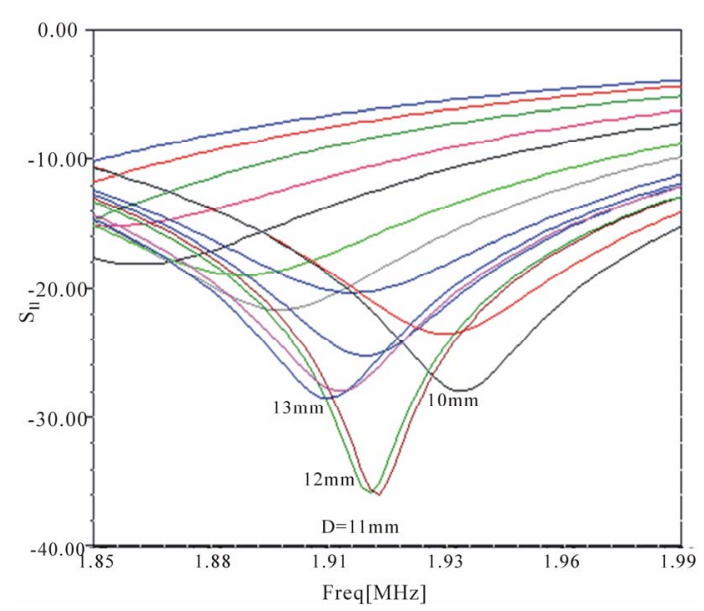

Figure 5. Parametric simulation by varying $D$.

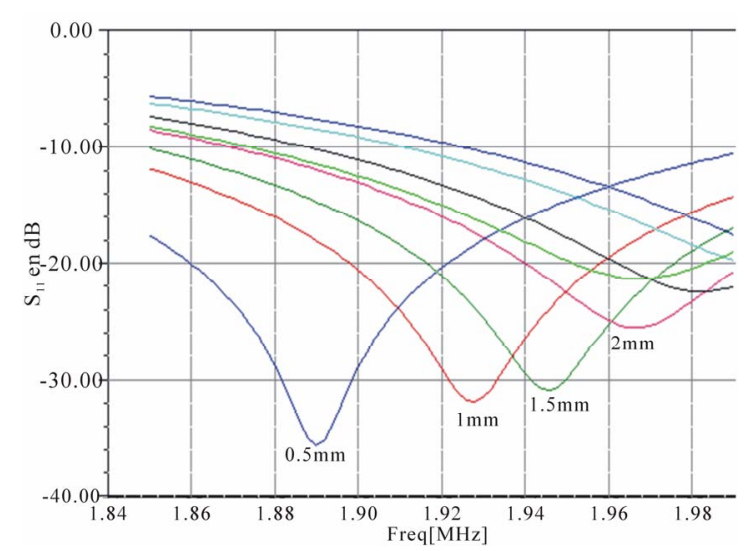

Figure 6. Parametric simulation by varying $W_{s}($ step $=0.5$ mm).

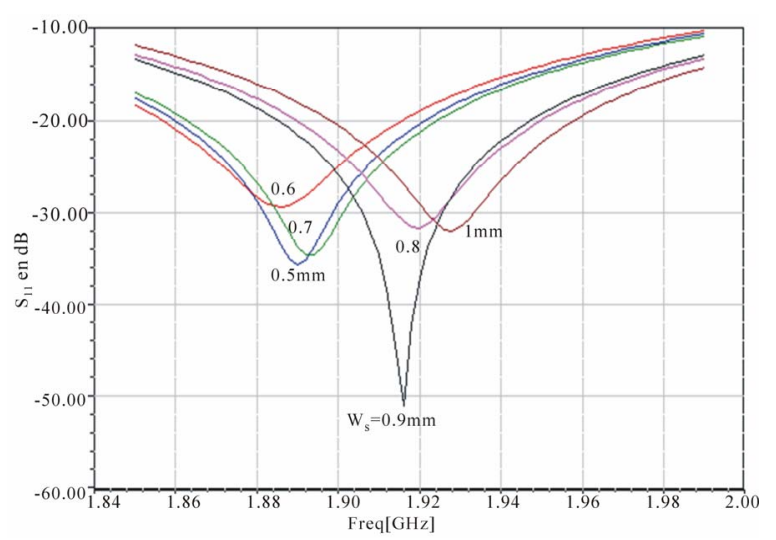

Figure 7. Parametric simulation by varying $W_{s}($ step $=0.1$ mm).

value of $0.9 \mathrm{~mm}$. For all studied parameters, any linear relation can't be established between bandwidth enhancement and the parameter variation. We have then chosen the parameters $h, L_{g}, W_{g}, D$ and $W_{s}$. We can now look for the effect of the feeding point position $p$ to enhance the bandwidth or the input impedance.

\subsection{The Choice of the Feeding Point Position $p$}

The feeding point position $p$ is calculated from the rear edge of the patch. We will vary the $p$ parameter from 2 $\mathrm{mm}$ to $10 \mathrm{~mm}$ we mean all possible cases. The values 1 and $11 \mathrm{~mm}$ can't be taken because the feeding point is theoretically a circle that has a radius. The result is shown on Figure 8. We can consider the peak for $p=4$ $\mathrm{mm}$ an interesting position for feeding.

\section{The Designed Antenna Characterization}

The dimensions of the designed antenna for the PCS band are summarized in the Table 1. We will now present the antenna characteristics.

\subsection{The Reflection Loss}

The Figure 9 shows the $S_{11}$ parameter function of frequency in [0 - $3 \mathrm{GHz}$. We can see a peak of $S_{11}$ parameter around $1924 \mathrm{MHz}$ very close to the PCS central frequency $1920 \mathrm{MHz}$. Also, the $S_{11}$ values out of the PCS band are near 0 that means the designed antenna can't interfere with other radiations. We can also run the si-

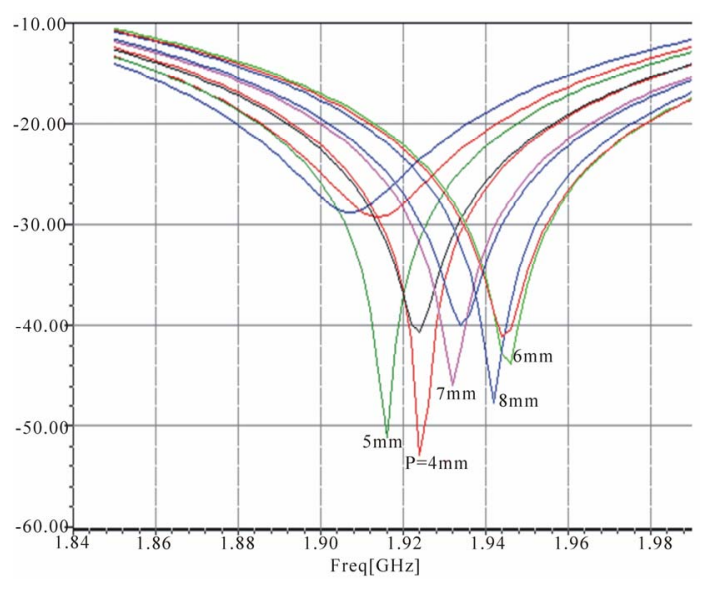

Figure 8. Parametric simulation by varying $p$.

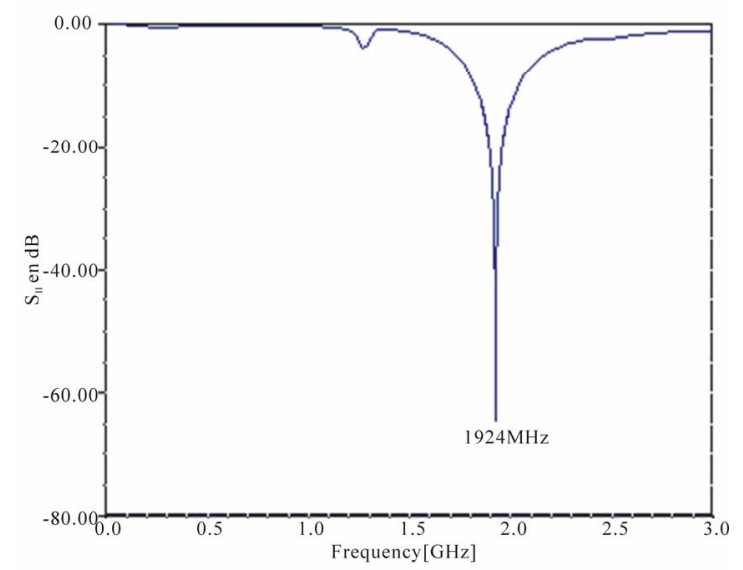

Figure 9. $S_{11}$ depending on frequency for [0 - $\left.3 \mathrm{GHz}\right]$. 
mulation by refining the sweep interval for more precision and as shown in Figure 10. The Figure 11 furnished by the simulation gives exactly $S_{11}=-12.43 \mathrm{~dB}$ for 1850 $\mathrm{MHz}$ (the low frequency of the PCS band), $S_{11}=-60 \mathrm{~dB}$ for $1925 \mathrm{MHz}$ (the peak frequency), the simulation tables give exactly a peak of $-64.46 \mathrm{~dB}$ for the frequency 1924.7 MHz and $S_{11}=-12.49 \mathrm{~dB}$ for $1990 \mathrm{MHz}$ (the high frequency of the PCS band). For the whole PCS band, the designed antenna has a $S_{11}$ litter than $-12.4 \mathrm{~dB}$. It is very interesting result.

\subsection{The VSWR and Bandwidth}

We obtain as shown in Figure 12(a) VSWR $=1.62$ for $1850 \mathrm{MHz}$ (the lowest frequency of the PCS band), $\mathrm{VSWR}_{\min }=1.001$ for $1924.7 \mathrm{MHz}$ (the peak frequency), VSWR $=1.49$ for $1990 \mathrm{MHz}$ (the highest frequency of the PCS band). The VSWR is at its minimum, it's a very interesting result. Also, the PCS bandwidth (140 MHz) is for the designed antenna a 1:1.62 VSWR bandwidth. It is considered a very interesting result.

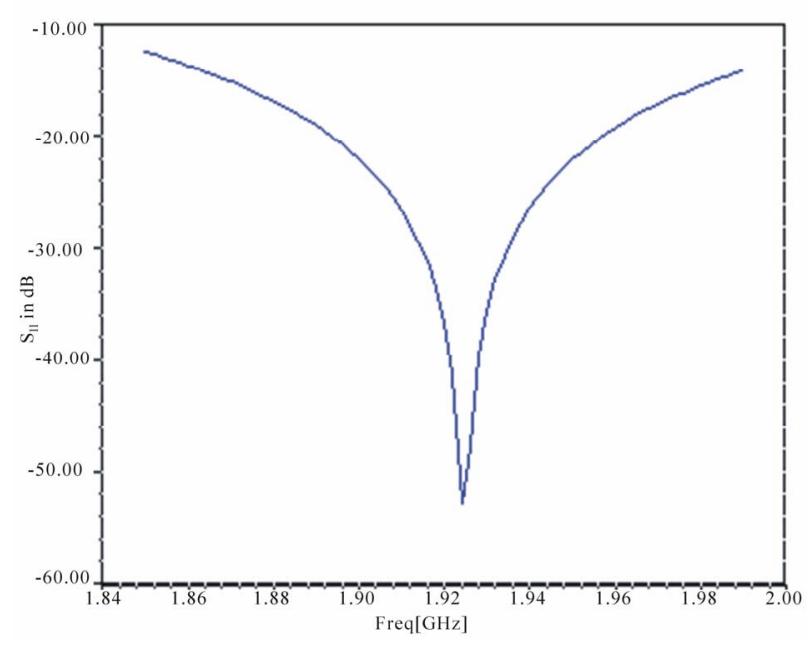

Figure 10. $S_{11}$ depending on frequency for PCS band.

Table 1. The designed antenna dimensions.

\begin{tabular}{cc}
\hline Antenna parameter & Value of the parameter $(\mathrm{mm})$ \\
\hline Patch length $L_{p}$ & 11 \\
Patch width $W_{p}$ & 30 \\
Ground plan length $L_{g}$ & 108 \\
Ground plan width $W_{g}$ & 45 \\
Height $h$ & 10 \\
Short plate width $W_{s}$ & 0.9 \\
Short plate position $D$ & 11 \\
Feeding point position & 4 \\
\hline
\end{tabular}

The designed antenna presents a 1:1.5 VSWR bandwidth equal to $130 \mathrm{MHz}$.

\subsection{The Smith Chart}

The Figure 13 shows a regular smith chart with interesting parameters of reflection, impedance, VSWR, and Q. The feeding point impedance for extremes and central frequencies are given in Table 2.

\subsection{Antenna Parameters}

The simulations results shown in Figure 14 give the antenna parameters. The obtained gain $G$ is $1.16 \mathrm{dBi}$ and the radiation efficiency is 1.0085 .

\subsection{The Diagram Pattern}

We can confirm by the Figure 15 that $(x z)$ is the E-plane and its maximum is for ( $\mathrm{phi}=0 \mathrm{deg}$ et theta $=56 \mathrm{deg}$ ). Also, we can verify that via the $3 \mathrm{D}$ polar diagram shown in Figure 16.

\begin{tabular}{|l|l|l|}
\hline \hline 31 & 1.924500 & -61.004117 \\
\hline 32 & 1.924550 & -62.153483 \\
\hline 33 & 1.924600 & -63.233862 \\
\hline 34 & 1.924650 & -64.076436 \\
\hline 35 & 1.924700 & -64.463742 \\
\hline 36 & 1.924750 & -64.262188 \\
\hline 37 & 1.924800 & -63.544375 \\
\hline 38 & 1.924850 & -62.519234 \\
\hline 39 & 1.924900 & -61.379179 \\
\hline 40 & 1.924950 & -60.239626 \\
\hline
\end{tabular}

Figure 11. The resonant frequency.

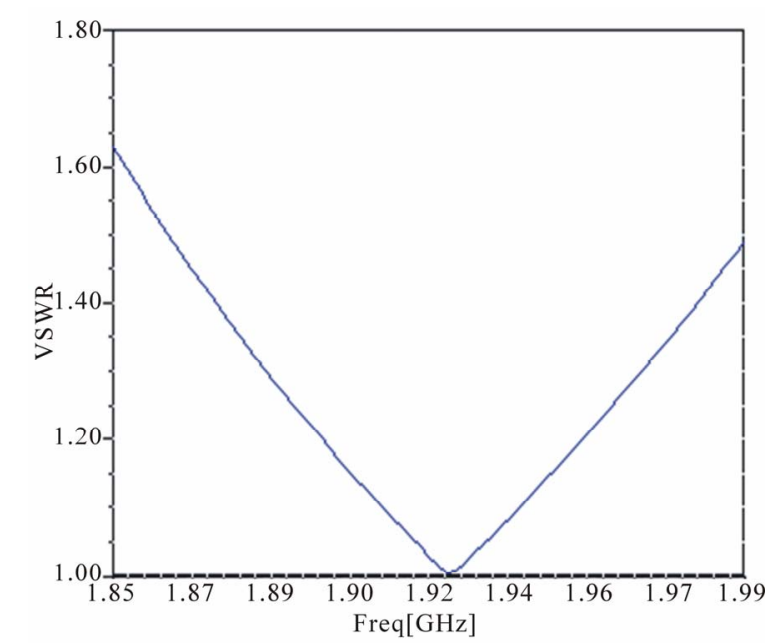

Figure 12. The VSWR depending on the frequency for the PCS band. 


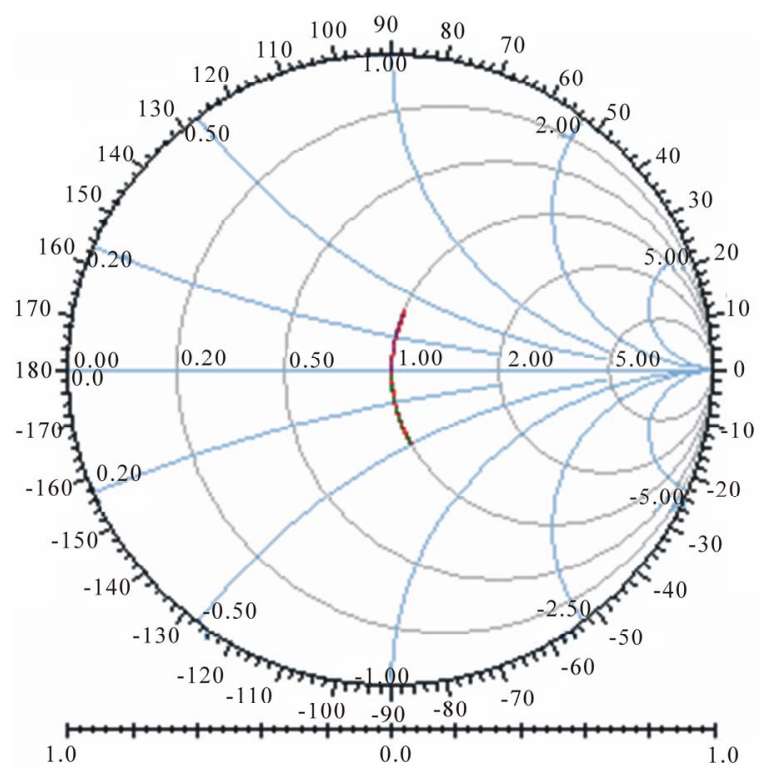

Figure 13. The smith chart for PCS band.

\begin{tabular}{|c|c|c|}
\hline \multicolumn{3}{|l|}{ Antenna parameters: } \\
\hline Quantity & Value & Units \\
\hline $\operatorname{Max} U$ & 0.92134 & W/sr \\
\hline Peak Directivity & 1.1483 & \\
\hline Peak Gain & 1.1581 & \\
\hline Peak Realized Gain & 1.1578 & \\
\hline Radiated Power & 1.0083 & W \\
\hline Accepted Power & 0.99978 & W \\
\hline Incident Power & 1 & $\mathrm{~W}$ \\
\hline Radiation Efficiency & 1.0085 & \\
\hline Decay Factor & 0 & \\
\hline
\end{tabular}

Figure 14. Antenna parameters as given by HFSS.

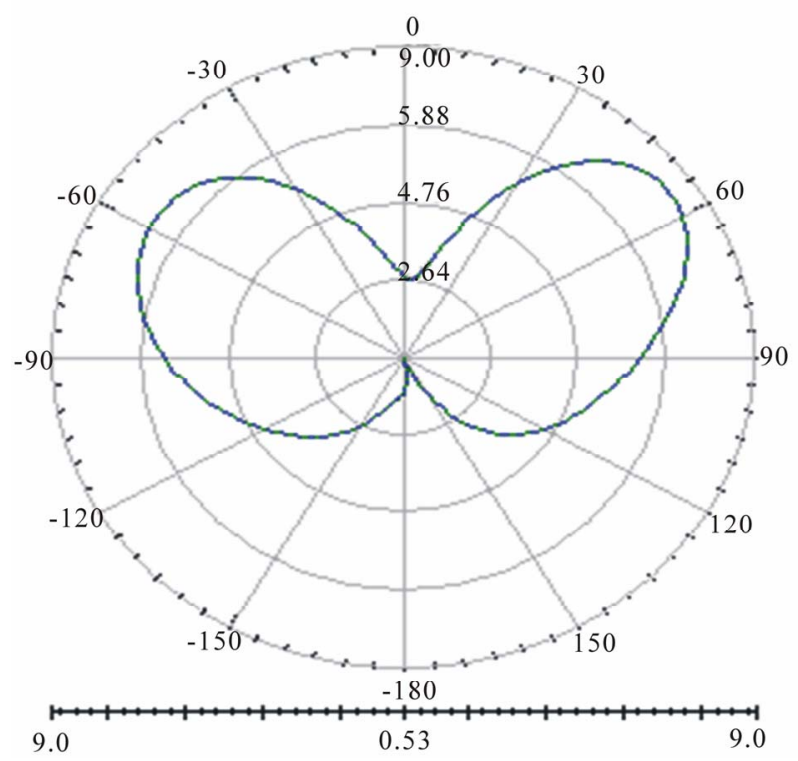

Figure 15. The E-field diagram pattern (for phi $=0 \mathrm{deg}$ ).
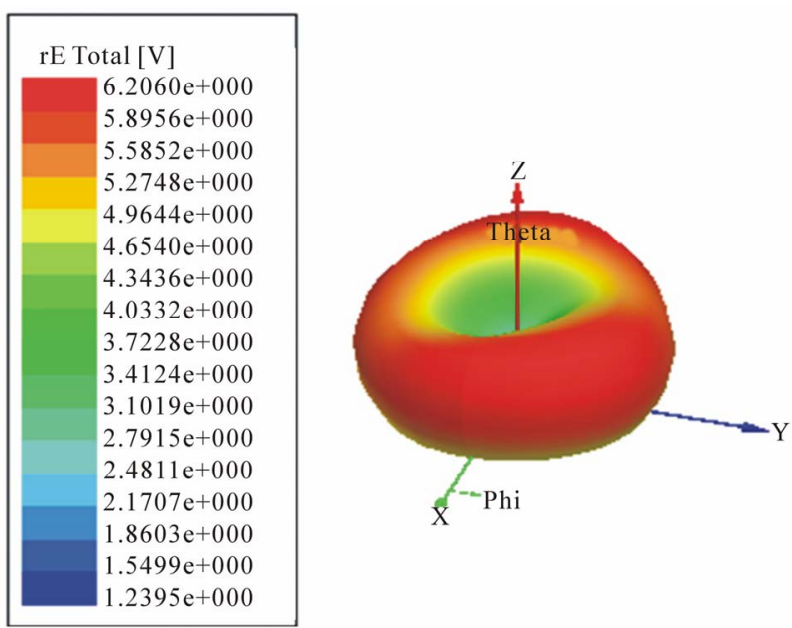

Figure 16. The E-field 3D polar diagram pattern.

Table 2. The impedances table in PCS band.

\begin{tabular}{ccc}
\hline $\begin{array}{c}\text { Frequency } \\
(\mathrm{MHz})\end{array}$ & $\begin{array}{c}\text { Impedance } Z \\
\text { (Magnitude en } \Omega, \text { Phase) }\end{array}$ & $\begin{array}{c}\text { Port } Z_{0} \\
\text { (Magnitude en } \Omega \text {, Phase) }\end{array}$ \\
\hline 1850 & $(39.592,-26.1)$ & $(35.239,0)$ \\
1924.7 & $(35.281,0)$ & $(35.239,0)$ \\
1990 & $(38.438,21.5)$ & $(35.239,0)$ \\
\hline
\end{tabular}

\section{Conclusions}

To design a PIFA for PCS band, we followed a methodology based on parametric simulations. During the design process, we can make some conclusions:

1) The PIFA characteristics are affected by each of the changed parameter (the height of the radiating plate, the ground plane dimensions, the position and width of the short plate, the position of the feeding point).

2) When we work in a medium bandwidth (like our case $140 \mathrm{MHz}$ ), any linear relation can't be established between the way the characteristics of the antenna change and the way the parameter change. The parametric simulations are very interesting to make effective choice.

3) It's interesting in the design of the PIFA to search an effective solution not the optimal one. In fact, difference will be very little, and also the values of the PIFA dimensions must be practical not theoretical. The important fact is that the solution respects the requirements.

4) In comparison with precedent designed PIFA [4,5] for different frequency bands, the antenna presents a very high radiation efficiency and larger bandwidth.

5) The designed PIFA for the PCS use respects the requirements especially for the resonant frequency, the VSWR, the bandwidth, the reflection loss, the anisotropy and the miniaturization.

The Table 3 summarizes the characteristics and dimensions of the designed antenna. The analysis of those 
Table 3. The design antenna parameters and dimensions.

\begin{tabular}{cc}
\hline Antenna parameter & Value of the parameter \\
\hline Patch Length $L_{p}$ & $11 \mathrm{~mm}$ \\
Patch width $W_{p}$ & $30 \mathrm{~mm}$ \\
Ground plan length $L_{g}$ & $108 \mathrm{~mm}$ \\
Ground plan width $W_{g}$ & $45 \mathrm{~mm}$ \\
Height $h$ & $10 \mathrm{~mm}$ \\
Short plate width $W_{s}$ & $0.9 \mathrm{~mm}$ \\
Short plate position $D$ & $11 \mathrm{~mm}$ \\
Feeding point position & $4 \mathrm{~mm}$ \\
Resonnat frequency & $1924.7 \mathrm{MHz}$ \\
$S_{11}$ in 1850 MHz & $-12.43 \mathrm{~dB}$ \\
$S_{11}$ in 1924.7 MHz & $-64.5 \mathrm{~dB}$ \\
$S_{11}$ in 1990 MHz & $-12.49 \mathrm{~dB}$ \\
VSWR $\min$ & 1.001 \\
$1: 1.5$ VSWR bandwith & $130 \mathrm{MHz}$ \\
$1: 1.62$ VSWR bandwith & $140 \mathrm{MHz}(\mathrm{PCS}$ band) \\
Gain & $1.16 \mathrm{~dB}$ \\
Radiation efficiency & 1.009 \\
E total max & $0 \mathrm{deg}$, theta $=56 \mathrm{deg}$ \\
\hline
\end{tabular}

results makes from our designed antenna a succeeded trade-off that respects PCS requirements.

\section{REFERENCES}

[1] K. L. Wong, "Introduction and Overview," In: K. L. Wong, Ed., Planar Antennas for Wireless Communications, John Willy and Sons, Hoboken, 2003, p. 1.

[2] K. L. Virga and Y. Rahmat-samii, "Low-Profile Enhanced Bandwith PIFA for Wireless Communications Packaging," IEEE Transactions on Microwave Theory and Techniques, Vol. 45, No. 10, 1997, pp. 1879-1888. doi:10.1109/22.641786

[3] W.-J. Liao, T.-M. Liu and S.-Y. Ho, "Miniaturized PIFA Antenna for $2.4 \mathrm{GHz}$ ISM Band Applications," IEEE Proceedings of the 6th European Conference on Antennas and Propagation (EUCAP), Prague, 26-30 March 2012, pp. 3034-3037.

[4] A. K. Skrivervik, J.-F. Zürcher, O. Staub and J. R. Mosig, "PCS Antenna Design: The Challenge of Miniaturization," IEEE Antennas and Propagation Magazine, Vol. 43, No. 4, 2001, pp. 12-27. doi:10.1109/74.951556

[5] Ansoft Corporation, "HFSS 10.0 User's Guide," Ansoft Corporation, Pittsburg, 2005.

[6] J. McClure, "Statistics for Microarrays: Design, Analysis, and Inference," Rev.1.06, Ansoft Corporation, Pittsburg, 2005 . 